\title{
Changes in preoperative endoscopic and percutaneous bile drainage in patients with periampullary cancer undergoing pancreaticoduodenectomy in Ontario: effect on clinical practice of a randomized trial
}

\author{
D.J. Kagedan $\mathrm{MD} \mathrm{MSc}^{*}$ J.D. Mosko MD MSc, ${ }^{\dagger \neq}$ M.E. Dixon $\mathrm{MD}^{*}{ }^{*}$ P.J. Karanicolas MD PhD, ${ }^{* \neq \S}$ \\ A.C. Wei MD MSc, ${ }^{* \neq \| \# \# ~ N . ~ G o y e r t ~ M A, ~}{ }^{\S}$ Q. Li MMath, ${ }^{* *}$ N. Mittmann $\mathrm{PhD}_{1}^{+\dagger}$ and N.G. Coburn MD MPH${ }^{* \neq \S \|}$
}

\section{ABSTRACT}

Background In 2010, a multicentre randomized controlled trial reported increased postoperative complications in pancreaticoduodenectomy (PDE) patients undergoing preoperative biliary decompression (PBD). We evaluated the effect of that publication on rates of PBD at the population level.

Methods This retrospective observational cohort study identified patients undergoing PDE for malignancy, 2005-2013, linking them with administrative health care databases covering medical services for a population of 13.5 million. Patients undergoing PBD within 6 weeks before their surgery were identified using physician billing codes and were divided into those undergoing PDE before and after article publication, with a 6-month washout period. Chi-square tests were used to compare rates of PBD.

Results Of 1997 PDE patients identified, 963 underwent surgery before article publication, and 911, after (123 during the washout period). The rate of PBD was $47.5 \%$ before publication, and $41.6 \%$ after $(p=0.01)$. The lowest PBD rates occurred immediately after publication, in 2010 and 2011. Similar results were observed when the cohort was restricted to patients seen preoperatively by a gastroenterologist $(n=1412)$.

Conclusions Rates of PвD have declined a small, but significant, amount after randomized trial publication. Persistence of PBD might relate to suboptimal knowledge translation, the role of PBD in diagnosis of periampullary malignancy, and treatment of complications (cholangitis, severe hyperbilirubinemia) or anticipation of delay from diagnosis to surgery. The nadir in PBD rates after article publication and the subsequent rise suggest an element of transience in the effect of article publication on clinical practice. Further investigation into the reasons for persistent PBD is needed.

Key Words Pancreatic neoplasia, resection, periampullary tumours, outcomes

Curr Oncol. 2018 Oct;25(5):e430-e435

www.current-oncology.com

\section{INTRODUCTION}

Malignant tumours of the periampullary region often obstruct biliary drainage as they enlarge, resulting in hyperbilirubinemia and jaundice at presentation in $47 \%-90 \%$ of patients ${ }^{1-3}$. Substantial controversy has arisen about the benefits of preoperative interventions to decompress the blocked biliary ducts and relieve jaundice before pancreaticoduodenectomy (PDE) in patients with tumours amenable to surgical resection ${ }^{4}$. Obstructive jaundice has been hypothesized to impair hemostasis, immune function, and wound healing; preoperative biliary decompression (PBD) before PDE has therefore been routinely performed for many years ${ }^{5-9}$. However, mounting 
evidence suggests that, contrary to its theoretical benefits, routine PBD of jaundiced patients is associated with increased morbidity-specifically, increased infectious complications ${ }^{1,2,10-13}$.

In January 2010, a multicentre prospective randomized controlled trial (RCT) published by van der Gaag et al. ${ }^{14}$ in the New England Journal of Medicine reported that morbidity was increased in patients undergoing PBD compared with patients who proceeded directly to surgery. The DROP trial randomized 202 jaundiced patients with resectable cancers of the pancreatic head and no evidence of cholangitis either directly to surgery (PDE within 1 week of diagnosis) or to PBD (biliary drainage for 4-6 weeks, followed by PDE) ${ }^{14,15}$. In the PBD group, patients experienced nearly double the rate of serious complications [74\% vs. $39 \%$; relative risk: $0.54 ; 95 \%$ confidence interval (CI): 0.41 to 0.71 ] seen in the direct-to-surgery group. Infectious complications (wound infection, pneumonia, cholangitis) in particular were more common in the PBD patients. The authors concluded that routine PBD led to increased complications and did not confer any benefit with respect to outcomes ${ }^{14}$. The study provided level I evidence favouring direct surgery over $\mathrm{PBD}^{16}$. To ascertain the impact of level I evidence on actual clinical practice, we assessed the effect of the DROP study on rates of PBD before PDE by comparing PBD rates at the population level before and after publication of the RCT.

\section{METHODS}

This population-based observational cohort study used administrative health care data from databases held at the Institute for Clinical Evaluative Sciences (ICES) that were linked using unique patient identifiers. The linked databases included the Discharge Abstract Database maintained by the Canadian Institute for Health Information, the oHIP (Ontario Health Insurance Plan) database, the Registered Persons Database, and the Ontario Cancer Registry. The Discharge Abstract Database contains dates and details of all hospital admissions of more than 24 hours' duration in the province ${ }^{17}$. The oHIP database contains dates of all medically necessary consultations and interventions billed by a physician in the province (including those related to periampullary malignancy). The Registered Persons Database contains patient sociodemographic information, and the Ontario Cancer Registry contains histopathologic details for all oncologic resection specimens in Ontario.

Patients undergoing PDE in the province of Ontario (population 13.5 million $^{18}$ ) between 1 April 2005 and 30 September 2013 were identified from discharge records in the Discharge Abstract Database (Canadian Classification of Health Interventions codes 1.OK.87 and 1.OK.91), as previously described ${ }^{19}$. The cohort was further restricted to patients diagnosed with a periampullary malignancy within 6 months of undergoing PDE. Periampullary malignancy was defined using the International Classification of Diseases for Oncology (3rd edition) topography and histology or behavior codes contained in the Ontario Cancer Registry database. Patients treated with neoadjuvant chemotherapy (defined using oHIP billing codes for administration of chemotherapy in the 365 days before PDE) were excluded from the analysis.
The primary outcome of interest, PBD, was identified using OHIP billing codes for endoscopic retrograde cholangiopancreatography (Z558, Z561, and Z760), percutaneous transhepatic cholangiography (J013 and S233), and bile duct intubation for obstruction (Z542). In accordance with the definition used by van der Gaag et al. in their study, PBD was defined as occurring within 42 days (6 weeks) before the date of the PDE surgery. Preoperative biliary decompression was defined as a binary categorical variable, in that each patient either underwent PBD or did not; patients who underwent multiple PBD procedures were not distinguished.

To ascertain the effect of the publication of the van der Gaag et al. multicentre RCT recommending against PBD before PDE, the patient cohort was split into a pre-publication group and a post-publication group, with a 6-month washout period surrounding publication of the RCT results in January 2010. The pre-publication group underwent PDE between 1 April 2005 and 30 September 2009, and the post-publication group underwent PDE between 1 April 2010 and 30 September 2013. Chi-square tests were used to compare unadjusted rates of PBD between the pre- and post-publication groups, and between the pre-publication, post-publication, and washout groups. Rates of PBD were also stratified by year of PDE surgery and compared by chisquare test. A sensitivity analysis was performed restricting the cohort to patients who were seen in preoperative consultation by a gastroenterologist (identified using oHIP codes A415, C415, A135, and C135) within 100 days before surgery.

Logistic regression with backward elimination of covariates at $p>0.2$ was used to identify independent predictors of PBD, with adjustment for these covariates: time period, age, sex, comorbidity, socioeconomic status ${ }^{20,21}$. Age, sex, and socioeconomic status were defined using data from the Registered Persons Database. Geographic location of primary residence was used to define socioeconomic status, representing both rural or urban location and median neighbourhood income relative to other incomes in the geographic region (categorized from lowest to highest income quintile) based on census data, as previously described $^{22}$. To evaluate rurality while avoiding collinearity with median income, a hybrid variable termed "socioeconomic status" was created, wherein all rural patients were grouped into a single category ${ }^{23}$. Comorbidity burden was defined using the Johns Hopkins (Baltimore, MD, U.S.A.) Adjusted Clinical Groups system, based on the International Classification of Diseases (revision 10) diagnostic codes for each patient in the preceding 24 months; codes are then summed to generate a score from 0 to 32 (increasing values representing increasing comorbidity burden). To optimize model stability, a two-level categorical version of the variable with a cut-point of 10 was used as has previously been described $^{20,21}$. Results are presented as adjusted odds ratios (ORs) for undergoing PBD, with 95\% confidence intervals (CIs). Two-sided hypothesis testing was performed, and an alpha of 0.05 was used to establish statistical significance.

All analyses were performed at ICES using the SAS software application (version 9.2: SAS Institute, Cary, NC, U.S.A.). Appropriate research ethics board approval for this project was obtained at Sunnybrook Health Sciences Centre and at ICES. 


\section{RESULTS}

From 2005 to 2013, 2660 patients with linkable unique identifiers underwent PDE in Ontario (26 patients were excluded because of missing identifiers). Of those 2660 patients, 2053 had a diagnosis of periampullary malignancy. The 56 patients who received neoadjuvant therapy were excluded. The remaining 1997 patients formed the cohort subject to analysis. Based on date of surgery, that cohort was then subdivided into a pre-publication group ( $n=963$, PDE occurring before 1 October 2009), a post-publication group ( $n=911$, PDE occurring after 31 March 2010), and a washout group ( $n=123$, PDE occurring between 1 October 2009 and 31 March 2010). Table I presents the baseline clinical and sociodemographic characteristics of those groups (8 patients excluded because of missing socioeconomic status).

Of the 1997 periampullary cancer patients who underwent PDE, 886 underwent PBD within the 6 weeks before surgery $(44.4 \%)$. A significant decline in the rate of PBD use was observed from the pre-publication group $(47.5 \%)$ to the post-publication group $(41.6 \%, p=0.011$, Figure 1). A similar decline in the annual rate of PBD was also observed, to $40.2 \%$ in 2013 from $52.5 \%$ in 2005 ( $p=0.05$, Figure 2). Interestingly, the lowest annual rates of PBD were observed in 2010 (39.3\%) and 2011 (38.7\%), immediately

TABLE I Clinical characteristics of 1997 patients undergoing pancreaticoduodenectomy for malignant indication in the periods before and after the publication of van der Gaag et al., 2010 ${ }^{14}$, with a 6-month washout period

\begin{tabular}{|c|c|c|c|}
\hline \multirow[t]{2}{*}{ Variable } & \multicolumn{3}{|c|}{ Relation of patient group to publication } \\
\hline & Before & Washout & After \\
\hline Patients (n) & 963 & 123 & 911 \\
\hline \multicolumn{4}{|l|}{ Age group } \\
\hline$\leq 60$ Years & 323 & 33 & 278 \\
\hline $61-70$ Years & 313 & 47 & 319 \\
\hline 71-80 Years & 283 & 34 & 259 \\
\hline$\geq 81$ Years & 44 & 9 & 55 \\
\hline \multicolumn{4}{|l|}{ Sex } \\
\hline Women & 421 & 51 & 380 \\
\hline Men & 542 & 72 & 531 \\
\hline \multicolumn{4}{|c|}{$\begin{array}{l}\text { Comorbidity group } \\
\text { (by score on the } \mathrm{ACG}^{\mathrm{a}} \text { ) }\end{array}$} \\
\hline $0-9$ & 372 & 51 & 357 \\
\hline $10-32$ & 591 & 72 & 554 \\
\hline \multicolumn{4}{|c|}{ Socioeconomic status ${ }^{b}$} \\
\hline Urban 5 & 193 & 26 & 187 \\
\hline Urban 4 & 165 & 24 & 160 \\
\hline Urban 3 & 175 & 20 & 137 \\
\hline Urban 2 & 161 & 33 & 163 \\
\hline Urban 1 & 136 & 12 & 142 \\
\hline Rural & 130 & 8 & 117 \\
\hline
\end{tabular}

after publication of the RCT. Those findings persisted when the cohort was restricted to patients seen preoperatively by a gastroenterologist ( $n=1412$ ), with $54.2 \%$ of patients undergoing PBD in 2005, declining to $43.8 \%$ of patients in 2013 ( $p=0.16$, Figure 3).

After multivariate logistic regression adjusting for covariates, the likelihood of undergoing PBD was found to be significantly lower for patients in the post-publication

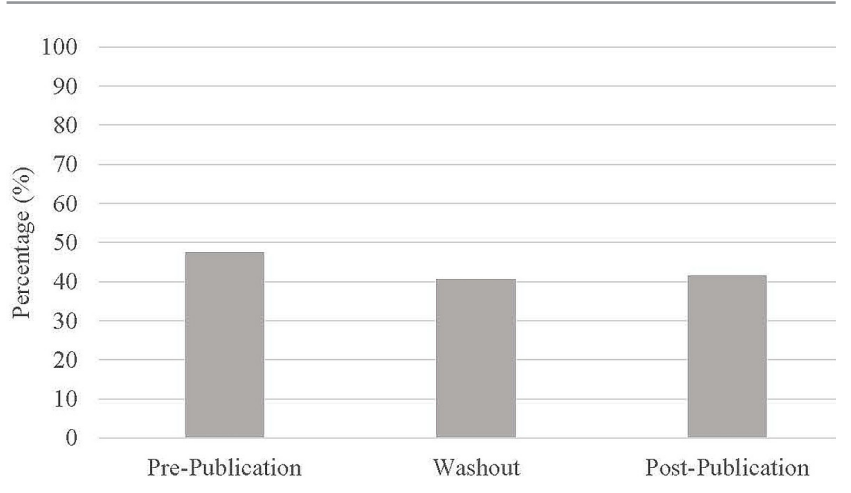

FIGURE 1 Rates of preoperative biliary drainage in 1997 patients undergoing pancreaticoduodenectomy for cancer in the pre-publication, washout, and post-publication periods $(p=0.03)$.

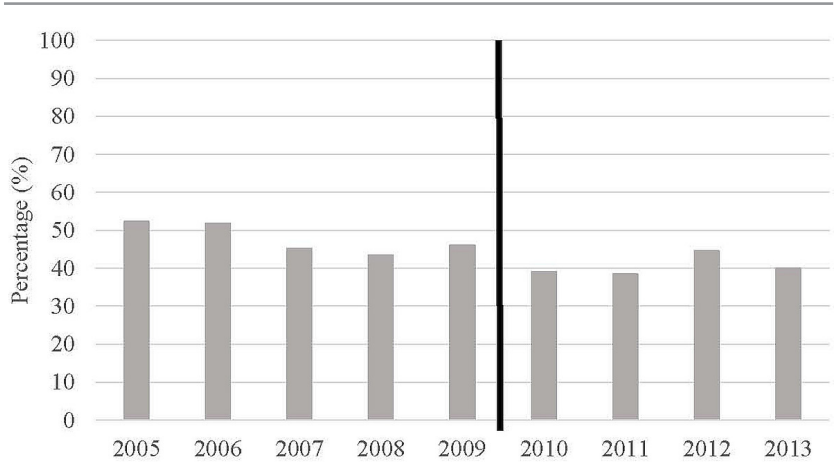

FIGURE 2 Rates of preoperative biliary drainage in 1997 patients undergoing pancreaticoduodenectomy for cancer by year of surgery $(p=0.05)$. The vertical line denotes article publication.

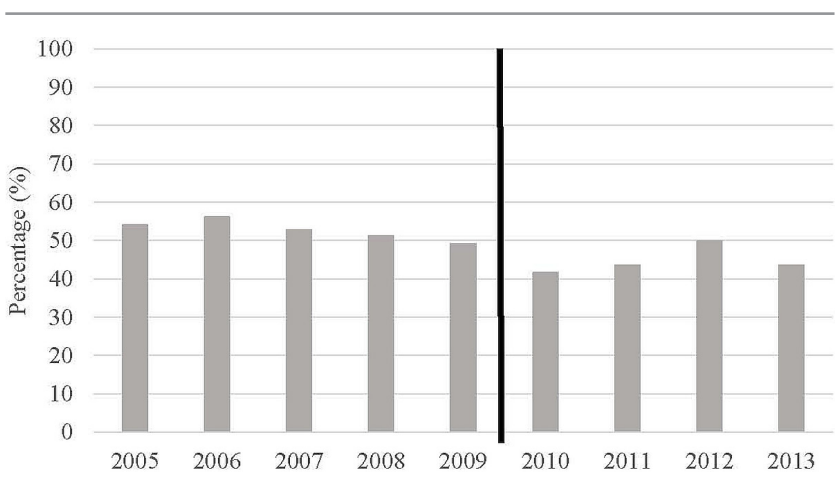

FIGURE 3 Rates of preoperative biliary drainage in 1412 patients undergoing pancreaticoduodenectomy for cancer who were seen preoperatively by a gastroenterologist $(p=0.16)$. The vertical line denotes article publication. 
period than for patients in the pre-publication period (oR: 0.794; 95\% cı: 0.661 to 0.955 ; Table II). Additionally, compared with patients living in the highest-income urban neighbourhoods, those living in the lowest-income urban neighbourhoods had a significantly lower likelihood of undergoing PBD (OR: 0.677; 95\% CI: 0.497 to 0.922). Living in a rural area was not associated with undergoing PBD (oR: 0.843 ; 95\% CI: 0.614 to 1.157 ). An association of PBD with age $(p=0.850)$, sex ( $p=0.652)$, or comorbidity burden $(p=0.557)$ was not observed.

\section{DISCUSSION}

In the present study, we compared rates of PBD before PDE for periampullary cancer before and after publication of a multicentre RCT demonstrating inferior outcomes with PBD, identifying a decline to $41.6 \%$ after article publication from $47.5 \%$ before publication. Previously published single- and multi-institutional studies have reported PBD rates ranging from $40 \%$ to $72 \%{ }^{9,12,24-27}$. The decline in PBD rates after publication of the van der Gaag et al. RCT is small, but convincingly present. Although the reduction in PBD might be related to secular trends and a general tendency away from routine PBD, the putative influence of van der Gaag's article is underscored by the observation that the lowest rates of PBD occurred in 2010 and 2011, immediately after the article's publication. Furthermore, that nadir in PBD rates and the subsequent rise suggests an element of transience in the effect of article publication, wherein the effect on practice patterns is most pronounced immediately after publication, and then diminishes slightly over time. Other possible explanations for that observation include the subsequent publication of large single-institution series reporting no increase in overall morbidity associated with $\mathrm{PBD}^{28}$. Despite the inferior methodologic rigour of results

TABLE II Independent predictors of preoperative biliary drainage in 1989 patients by logistic regression ${ }^{\mathrm{a}}$

\begin{tabular}{lccc}
\hline \multirow{2}{*}{ Variable } & \multirow{2}{*}{$\begin{array}{c}\text { Adjusted } \\
\text { odds ratio }\end{array}$} & \multicolumn{2}{c}{$\mathbf{9 5 \%} \mathbf{C L}$} \\
\cline { 3 - 4 } & & Lower & Upper \\
\hline Time period & & & \\
Before & Reference & & \\
Washout & 0.745 & 0.508 & 1.093 \\
After & $\mathbf{0 . 7 9 4}$ & $\mathbf{0 . 6 6 1}$ & $\mathbf{0 . 9 5 5}$ \\
Socioeconomic status & & & \\
Urban 5 & Reference & & \\
Urban 4 & 0.994 & 0.746 & 1.325 \\
Urban 3 & 1.015 & 0.759 & 1.359 \\
Urban 2 & 0.949 & 0.712 & 1.263 \\
Urban 1 & $\mathbf{0 . 6 7 7}$ & $\mathbf{0 . 4 9 7}$ & $\mathbf{0 . 9 2 2}$ \\
Rural & 0.843 & 0.614 & 1.157 \\
\hline
\end{tabular}

a Using backward elimination of covariates at $p>0.2$ (eliminated: age, sex, comorbidity). Adjusted odds ratios, with 95\% confidence intervals are presented. Boldface type indicates significance. b In relation to the publication by van der Gaag et al., $2010^{14}$. $\mathrm{OR}=$ odds ratio; $\mathrm{CL}=$ confidence limits. emerging from retrospective series compared with those derived from a RCT, the results of those series might undermine the conclusions of van der Gaag et al. and contribute to clinical equipoise with respect to $\mathrm{PBD}^{14,28}$.

Although the reduction in the PBD rate after publication of the RCT was statistically significant, the magnitude of decrease was relatively small, and a substantial proportion of patients with periampullary malignancy were still undergoing PBD at the end of the study period. That situation might partly be attributable to a lack of effective knowledge translation and suboptimal uptake of evidence in clinical practice, but several other factors likely contributed to the persistence of $\mathrm{PBD}$ before $\mathrm{PDE}^{29}$. Patients presenting with painless jaundice might have undergone endoscopic retrograde cholangiopancreatography with stent insertion as an initial step in their work-up and, hence, were diagnosed with periampullary malignancy only once a PBD had been performed. Others might have required urgent PBD for cholangitis or severe hyperbilirubinemia.

The logistics of completing a preoperative work-up and scheduling an urgent PDE outside the auspices of a clinical trial can require a considerable amount of time, and anticipation of a delay from diagnosis to surgery could possibly be contributing to the persistence of $\mathrm{PBD}^{30}$. Initial referral of most patients to a gastroenterologist instead of a surgeon might further contribute to uncertainty about the time to PDE and bias practitioners toward performing PBD. Furthermore, physicians might possibly doubt the applicability of the RCT's findings to their clinical practice, given the challenges of performing a PDE within 1 week of diagnosis. One potential mechanism for addressing those barriers to evidence uptake is the implementation of health care policy designed to encourage early involvement of surgeons and to facilitate timely PDE. Notably, the longer time to surgery associated with PBD and resolution of jaundice might not be detrimental to patient outcomes. In fact, some data suggest that delay to surgery might confer a protective effect against mortality in the postoperative period, although the mechanism underlying that observation is unclear ${ }^{31}$.

Limitations of the present study include its retrospective observational design, with the resultant potential for selection bias and confounding by indication. Based on the administrative databases interrogated, it is not possible to know which patients in the cohort were jaundiced or cholangitic, or what their intended management plan was. However, the incidence of jaundice-and clear indications for PBD (cholangitis, severe hyperbilirubinemia)—would be expected to be stable over the study time period, and there is no reason to expect a change with publication of the van der Gaag et al. RCT. In addition, the definition of PBD was based on biliary instrumentation; patients who underwent unsuccessful PBD, or multiple attempts at PBD, were therefore not distinguished. However, PBD is, with multiple attempts, successful in more than $94 \%$ of patients, suggesting that almost all patients identified as having undergone PBD in the present study actually achieved biliary drainage ${ }^{14,32}$. Finally, the finding of a correlation between RCT publication and the observed slight decrease in PBD rates could potentially be spurious, the result of a type 1 error or confounding variables both known and unknown; accordingly, conclusions should be drawn with caution. 


\section{CONCLUSIONS}

In the present study, we used a large series of patients undergoing PDE for periampullary malignancy across a heterogeneous population of patients and providers to report on the uptake in clinical practice of high-level RCT evidence. The results demonstrate a significant decrease in the rate of $\mathrm{PBD}$ use after publication of a pertinent $\mathrm{RCT}$, although the magnitude of the decrease was relatively small. Numerous patients still undergo PBD before PDE. Putative contributing factors include established referral patterns and the time delay until surgery. Further investigation is needed into the reasons for performing PBD; into practice variation at the regional, institutional, and provider levels; and into barriers and facilitators to incorporation of best evidence into routine clinical practice. The potential for health care policy to improve the uptake of new evidence in this area should also be explored.

\section{ACKNOWLEDGMENTS}

This study and its results were shared in an oral presentation at the Society of Surgical Oncology Annual Cancer Symposium; Seattle, WA, U.S.A.; 15-18 March 2017; and as a poster at the Canadian Surgery Forum, Victoria, BC; 14-16 September 2017.

This study was funded by an operating grant from the Cancer Research Society and the Rob Lutterman Memorial Fund, 2013-2015. The principal investigator (NGC) is the Sherif and Mary Lou Hanna Chair in Surgical Oncology Research for salary and operating funds. All funding was used for collection, management, analysis, and interpretation of data. This study was supported by ICES, which is funded by an annual grant from the Ontario Ministry of Health and Long-Term Care (монцтс). The opinions, results, and conclusions reported in this paper are those of the authors and are independent from the funding sources. No endorsement by ICES or the Ontario MOHLTC is intended or should be inferred. Parts of this material are based on data and information compiled and provided by the Canadian Institute for Health Information (CIHI). However, the analyses, conclusions, opinions, and statements expressed herein are those of the authors and not necessarily those of CIHI. Parts of this material are based on data and information provided by Cancer Care Ontario (cco). The opinions, results, views, and conclusions reported in this paper are those of the authors and do not necessarily reflect those of cco. No endorsement by cco is intended or should be inferred.

\section{CONFLICT OF INTEREST DISCLOSURES}

We have read and understood Current Oncology's policy on conflicts of interest, and we declare that we have none.

\section{AUTHOR AFFILIATIONS}

*Division of General Surgery, Department of Surgery, 'Division of Gastroenterology, Department of Medicine, and ${ }^{\ddagger}$ Faculty of Medicine, University of Toronto, Toronto, ON; ${ }^{\circledR}$ Sunnybrook Health Sciences Centre, "Institute of Health Policy, Management, and Evaluation, "Princess Margaret Cancer Centre, University Health Network, ${ }^{* *}$ Institute for Clinical Evaluative Sciences, and ${ }^{\dagger \dagger}$ Health Outcomes and PharmacoEconomic Research Centre, Toronto, ON.

\section{REFERENCES}

1. Fang Y, Gurusamy KS, Wang Q, et al. Pre-operative biliary drainage for obstructive jaundice. Cochrane Database Syst Rev 2012;:CD005444.

2. van der Gaag NA, KloekJJ, de Castro SM, Busch OR, van Gulik TM, Gouma DJ. Preoperative biliary drainage in patients with obstructive jaundice: history and current status. J Gastrointest Surg 2009;13:814-20.

3. Bakkevold KE, Arnesjo B, Kambestad B. Carcinoma of the pancreas and papilla of Vater: presenting symptoms, signs, and diagnosis related to stage and tumour site. A prospective multicentre trial in 472 patients. Norwegian Pancreatic Cancer Trial. Scand J Gastroenterol 1992;27:317-25.

4. Chen Y, Ou G, Lian G, Luo H, Huang K, Huang Y. Effect of preoperative biliary drainage on complications following pancreatoduodenectomy: a meta-analysis. Medicine (Baltimore) 2015;94:e1199.

5. Grande L, Garcia-Valdecasas JC, Fuster J, Visa J, Pera C. Obstructive jaundice and wound healing. BrJSurg 1990;77:440-2.

6. Katz SC, Ryan K, Ahmed N, et al. Obstructive jaundice expands intrahepatic regulatory $\mathrm{T}$ cells, which impair liver $\mathrm{T}$ lymphocyte function but modulate liver cholestasis and fibrosis. J Immunol 2011;187:1150-6.

7. Papadopoulos V, Filippou D, Manolis E, Mimidis K. Haemostasis impairment in patients with obstructive jaundice. $J$ Gastrointestin Liver Dis 2007;16:177-86.

8. Moole H, Bechtold M, Puli SR. Efficacy of preoperative biliary drainage in malignant obstructive jaundice: a meta-analysis and systematic review. World J Surg Oncol 2016;14:182.

9. Coates JM, Beal SH, Russo JE, et al. Negligible effect of selective preoperative biliary drainage on perioperative resuscitation, morbidity, and mortality in patients undergoing pancreaticoduodenectomy. Arch Surg 2009;144:841-7.

10. Liu C, Lu JW, Du ZQ, Liu XM, Lv Y, Zhang XF. Association of preoperative biliary drainage with postoperative morbidity after pancreaticoduodenectomy. Gastroenterol Res Pract 2015;2015:796893.

11. Sugiyama H, Tsuyuguchi T, Sakai Y, et al. Current status of preoperative drainage for distal biliary obstruction. World $J$ Hepatol 2015;7:2171-6.

12. Pisters PW, Hudec WA, Hess KR, et al. Effect of preoperative biliary decompression on pancreaticoduodenectomyassociated morbidity in 300 consecutive patients. Ann Surg 2001;234:47-55.

13. Lai EC, Lau SH, Lau WY. The current status of preoperative biliary drainage for patients who receive pancreaticoduodenectomy for periampullary carcinoma: a comprehensive review. Surgeon 2014;12:290-6.

14. van der Gaag NA, Rauws EA, van Eijck CH, et al. Preoperative biliary drainage for cancer of the head of the pancreas. NEngl JMed 2010;362:129-37.

15. van der Gaag NA, de Castro SM, Rauws EA, et al. Preoperative biliary drainage for periampullary tumors causing obstructive jaundice; drainage vs. (direct) operation (DROP-Trial). BMC Surg 2007;7:3.

16. Baron TH, KozarekRA. Preoperative biliary stents in pancreatic cancer—proceed with caution. N Engl J Med 2010;362:170-2.

17. Schultz SE, Rothwell DM, Chen Z, Tu K. Identifying cases of congestive heart failure from administrative data: a validation study using primary care patient records. Chronic Dis Inj Can 2013;33:160-6.

18. Statistics Canada. 2016 Census of Population. Cat. no. 98400-X2016013. Ottawa, Ontario: Statistics Canada; 2017.

19. Kagedan DJ, Goyert N, Li Q, et al. The impact of increasing hospital volume on 90-day postoperative outcomes following pancreaticoduodenectomy. J Gastrointest Surg 2017;21:506-15.

20. Reid RJ, Roos NP, MacWilliam L, Frohlich N, Black C. Assessing population health care need using a claims-based ACG morbidity measure: a validation analysis in the Province of Manitoba. Health Serv Res 2002;37:1345-64.

21. Reid RJ, MacWilliam L, Verhulst L, Roos N, Atkinson M. Performance of the ACG case-mix system in two Canadian provinces. Med Care 2001;3:86-99. 
22. Mackillop WJ, Zhang-Salomons J, Groome PA, Paszat L, Holowaty E. Socioeconomic status and cancer survival in Ontario. J Clin Oncol 1997;15:1680-9.

23. Kagedan DJ, Abraham L, Goyert N, et al. Beyond the dollar: influence of sociodemographic marginalization on surgical resection, adjuvant therapy, and survival in patients with pancreatic cancer. Cancer 2016;122:3175-82.

24. Uemura K, Murakami Y, Satoi S, et al. Impact of preoperative biliary drainage on long-term survival in resected pancreatic ductal adenocarcinoma: a multicenter observational study. Ann Surg Oncol 2015;22(suppl 3):S1238-46.

25. Strom TJ, Klapman JB, Springett GM, et al. Comparative longterm outcomes of upfront resected pancreatic cancer after preoperative biliary drainage. Surg Endosc 2015;29:3273-81.

26. di Mola FF, Tavano F, Rago RR, et al. Influence of preoperative biliary drainage on surgical outcome after pancreaticoduodenectomy: single centre experience. Langenbecks Arch Surg 2014;399:649-57.

27. Sohn TA, Yeo CJ, Cameron JL, Pitt HA, Lillemoe KD. Do preoperative biliary stents increase postpancreaticoduodenectomy complications? J Gastrointest Surg 2000;4:258-67.
28. Sahora K, Morales-Oyarvide V, Ferrone C, et al. Preoperative biliary drainage does not increase major complications in pancreaticoduodenectomy: a large single center experience from the Massachusetts General Hospital. J Hepatobiliary Pancreat Sci 2016;23:181-7.

29. Davis D. Continuing education, guideline implementation, and the emerging transdisciplinary field of knowledge translation. J Contin Educ Health Prof 2006;26:5-12.

30. Iacono C, Ruzzenente A, Campagnaro T, Bortolasi L, Valdegamberi A, Guglielmi A. Role of preoperative biliary drainage in jaundiced patients who are candidates for pancreatoduodenectomy or hepatic resection: highlights and drawbacks. Ann Surg 2013;257:191-204.

31. Eshuis WJ, van der Gaag NA, Rauws EA, et al. Therapeutic delay and survival after surgery for cancer of the pancreatic head with or without preoperative biliary drainage. Ann Surg 2010;252:840-9.

32. Nakai Y, Isayama $\mathrm{H}$, Yamamoto $\mathrm{N}$, et al. Indications for endoscopic ultrasonography (EUs)-guided biliary intervention: does eus always come after failed endoscopic retrograde cholangiopancreatography? Dig Endosc 2017;29:218-25. 